FERMILAB-Pub-87/185-E

[E-741/CDF]

\title{
A Computer-Controlled Wire Tension Measurement System Used in the Fabrication of the CDF Central Drift Tube Array*
}

\author{
S. Bhadra, S. Errede $\star$ L. Fishback, H. Keutelian, and P. Schlabach \\ Loomis Laboratory of Physics \\ The University of Illinois at Urbana-Champaign \\ 1110 W. Green Street \\ Urbana, Illinois 61801 USA
}

July-August 1987

*Submitted to Nucl. Instrum. Methods A 


\title{
A Computer-Controlled Wire Tension
}

\section{Measurement System Used in the Fabrication of the CDF Central Drift Tube Array}

S. Bhadra, S. Errede*, L. Fishback, H. Keutelian, P. Schlabach

Loomis Laboratory of Physics

The University of Illinois at Urbana-Champaign

1110 W. Green Street

Urbana, Illinois, 61801 USA

August 4, 1987

\begin{abstract}
We have developed a sensitive computer-controlled method for measuring the anode wire tensions of individual drift tubes which have a wire support located mid-length along the tube. By resonating the anode wire at higher harmonics of the fundamental resonance frequency, simultaneous
\end{abstract}

-Alfred P. Sloan Research Fellow, 1985-89 
measurement of the anode wire tension and verification of the location of the wire support was achieved. The anode wire tension for each drift tube was measured to better than $0.5 \%$ accuracy and the location of the anode wire support measured to better than $0.01 \%$ for drift tubes 3 meters in length. This method was used for measuring the wire tensions of 2,226 drift tubes during the fabrication of the CDF Central Drift Tube array.

\section{Introduction}

During the fabrication of the CDF Central Drift Tube array [1] we developed a computer-controlled method for measuring the anode wire tensions of individual drift tubes made for the array. Measurements of drift tube anode wire tensions using this system could be done relatively quickly and easily, and with a high degree of accuracy and reproducability. Data taken with this wire tension measurement system were written to a fabrication database. Studies of personnel and time-dependent aspects associated with the wire-stringing process could then be performed, providing invaluable feedback to personnel during the wire-stringing stage of the fabrication of the CDF Central Drift Tube array.

Each drift tube in the 2,016-wire CDF Central Drift Tube (CDT) array consists of a $3048.000 \pm 0.050 \mathrm{~mm}$ long, $12.7 \mathrm{~mm}$ diameter, $200 \mu \mathrm{m}$ wall-thickness hard-drawn non-magnetic 304 stainless steel tube. Delrin endpin/feedthru assemblies at each end of the tube position and hold the $50 \mu \mathrm{m}$ diameter Stablohm800 non-magnetic stainless steel wire in place. In order to prevent electrostatic instabilities during $\mathrm{HV}$ operation of the CDT array, it was necessary to install 
a wire support in each drift tube, located mid-length along the tube. Because a region of the anode wire near the wire support is insensitive, drift tubes were made with one of four possible wire support locations so that a charged particle traversing the Central Drift Tube array in the region of the wire supports would encounter at most one such wire support [2].

\section{The CDF Central Drift Tube Wire Tension Measurement System}

Measurement of the wire tension of each tube was accomplished by passing a sinusoidal current $I(t)=I_{o} \sin \omega t, \omega=2 \pi f$ through the anode wire of the tube, which was placed in a magnetic field, $B$, oriented perpendicular to the anode wire. The Lorentz force, $\mathrm{dF}(\mathrm{t})=\mathrm{I}_{\mathrm{o}}$ BdLsinwt acting on a segment, $\mathrm{dL}$ of the wire produces a mechanical oscillation $y(t)=y_{o} \sin \omega t$. This induces an emf across the wire due to Faraday's law of electromagnetic induction, $\mathcal{E}(t)=\omega B d L y_{0} \cos \omega t$. The anode wire support, located near, but not precisely at the half-length distance of the tube, divides the total wire length, $L$, into two independent sections [3], of lengths $x$ and $L-x$. In general, on the $n^{\text {th }}$ harmonic of this system, standing waves occur at resonance frequencies $f_{1}$ and $f_{2}$ which are integer multiples of a half-wavelength; i.e. $\frac{n}{2} \lambda_{1}=x$ and $\frac{n}{2} \lambda_{2}=L-x$. The wave speed, $v=f_{1} \lambda_{1}=f_{2} \lambda_{2}$ is related to the anode wire tension, $T$, and mass

per unit length, $\mu$, of the wire by $v=\sqrt{\mathrm{T} / \mu}$. If $\mathrm{L}$ and $\mu$ are known [4], then by measurement of the resonance frequencies, $f_{1}$ and $f_{2}$, the anode wire tension, $T$, and wire support location, $x$, can be determined, since simultaneous solution 
of the two equations yields:

$T=\mu\left(\frac{2}{n}\right)^{2}\left(\frac{f_{1} f_{2}}{f_{1}+f_{2}}\right)^{2} L^{2}$ and $x=\left(\frac{f_{2}}{f_{1}+f_{2}}\right) L$ and $v=\left(\frac{2}{n}\right)\left(\frac{f_{1} f_{2}}{f_{1}+f_{2}}\right) L$

Note that $\mathrm{T}$ depends linearly on $\mu$, but varies as the square of the quantity $f_{1} f_{2} /\left(f_{1}+f_{2}\right)$ and as the square of the total anode wire length, $L$. The anode wire support position, $x$, is linearly proportional to $f_{2} /\left(f_{1}+f_{2}\right)$ and $L$. Although the location of the anode wire support position, $x$, for each tube was in principle known to an accuracy of better than $\pm 75 \mu \mathrm{m}$, we were concerned with detecting any and all possible failure modes associated with the drift tube fabrication process; hence the desire for a redundant check on this quantity.

The basic hardware for our wire tension measurement system followed along conceptual lines similar to that of ref. [5]. Fig. 1 shows the wire tension measurement system we used for measuring a total of 2,226 individual drift tubes fabricated for the CDF Central Drift Tube array. The set-up consisted of a Wavetek function generator, a Fluke frequency counter, a drift tube wire-tension measurement jig which held nine high-field permanent horseshoe magnets, a true rms AC to DC converter module, a DAC, a scanning ADC and a LSI-11/23 micro-computer interfaced to CAMAC and University of Illinois "Black-Box" electronics. The circuitry of the true rms AC to DC converter module is shown in Fig. 2.

The peak-to-peak amplitude of the Wavetek function generator is preset to a nominal value of $\sim 2.0 \mathrm{~V}$ (into a $50 \Omega$ load). The output of the 12-bit DAC, under computer control by the LSI-11/23, is applied to the VCG (voltage frequency 
control) input of the Wavetek function generator to vary its frequency. The frequency reponse of the Wavetek, as measured by the Fluke frequency counter, was linear with the applied DAC DC voltage to better than $0.1 \%$ over the full 4096-count range of the DAC and the frequency range of interest $(50-500 \mathrm{~Hz})$. After set-up and debugging of the system was completed, a 100:1 divider was installed to obtain a narrow frequency window over the full range of the DAC.

The true rms $\mathrm{AC}$ to $\mathrm{DC}$ converter module [6] measured the Wavetek input rms AC voltage and induced emf generated across the ends of the drift tube's resistive anode wire, converted it to a $\mathrm{DC}$ voltage which was then amplified, input to a 12-bit scanning ADC and then digitized. Typically, on the center of a resonance a signal height of $\sim 100 \mathrm{mV} \mathrm{DC}(\sim 100 \mathrm{ADC}$ counts $)$ was obtained at the output of the true rms AC to DC converter module. The digitized wire tension measurement data were displayed in real-time on a DEC VT-241 CG terminal and simultaneously written to a $30 \mathrm{MB}$ Winchester disk, on which the wire tension measurement fabrication database was located.

The $20 \mathrm{db}$ attenuator was used to obtain finer control of the output amplitude of the Wavetek function generator. The purpose of the $1000 \mu \mathrm{F}$ capacitor in series with the anode wire of the drift tube was merely to block any DC current component from the Wavetek function generator. The large value of the capacitance was to ensure flat frequency response down to well below the fundamental resonance frequency, $\sim 80 \mathrm{~Hz}$. The $4.0 \mathrm{~K} \Omega$ series resistor was used as a constant current source and to limit the current associated with the induced 
$e m f$, thus maintaining high intrinsic sensitivity for the signal. Great care was taken to achieve proper of shielding and grounding, due to the relatively high amplifier gain used in the system.

The anode wires of drift tubes for the CDT array were all strung at the same tension of 108.0 grams. The nominal anode wire resistance of strung drift tubes was typically $1988 \pm 3 \Omega[1]$. A drift tube to be wire-tension measured was inserted into the gaps between the field-shaping pole tips of all nine permanent magnets, which were bolted onto the aluminum $\mathrm{C}$-channel of the wire tension measuring jig. The drift tube was rigidly supported at $60 \mathrm{~cm}$ intervals by aluminum support clamps. The clamps gently locked the tube down into position during the wire tension measurement. The magnetic field between the field-shaping pole tips of the permanent magnets varied slightly from one magnet to another over the range of 2.1 to 2.5 kilo-gauss.

Due to the finite width of the resonances of the two fundamental frequencies, it was not possible to easily resolve the two resonances as distinct entities for all four types of tubes (as defined by the four locations of the wire supports). In one type of drift tube, the wire support was displaced by $12.70 \mathrm{~mm}$ from tube center towards the serial numbered end of the drift tube. For the three other types of drift tubes, the wire support was further displaced (in successive increments of $19.05 \mathrm{~mm}$ ) from the first type of drift tube wire support position. Much time was spent in the set-up and debugging stage studying the response of the wire tension measurement system on the fundamental harmonic all the way up to 
the $11^{\text {th }}$ harmonic. We finally chose to operate the system on the $5^{\text {th }}$ harmonic $(\sim 400 \mathrm{~Hz})$, where the two resonance curves were always well separated for each of the two halves of the tubes. The nominal widths of the resonances on the $5^{\text {th }}$ harmonic were typically $\sim 1.2 \mathrm{~Hz}$ (FWHM). This choice of harmonic also greatly simplified requirements for computer pattern-recognition and peak-finding code.

The nine magnets used in the drift tube wire tension measurement system were located in such a way as to optimize the generation of the $5^{\text {th }}$ harmonic. It was also possible to generate higher harmonics, but the limiting factor was the 10 cm width of the field-shaping pole faces of the magnets. The distance between two adjacent nodes should not be smaller than this width, since the Lorentz force acting on this segment of the anode wire and the resulting induced emf would both tend to cancel in this situation. Magnet locations were calculated as follows: The anode wire length was divided into two halves, and then divided again by a factor of five to obtain the distance between adjacent nodes of the generated $5^{\text {th }}$ harmonic standing wave $(\sim 30 \mathrm{~cm})$. The magnets were then placed at the midpoints between two nodes (i.e. at the antinodes) and their polarity (i.e. the direction of the magnetic field) reversed between adjacent magnets. Four magnets were placed on one side of the wire support and five magnets on the other side. This "spontaneous symmetry breaking" was deliberately done in order to systematically indicate the "polarity" of the tube, again as defined by the wire support location. The orientation of a tube as inserted into the wire tension measuring jig was always such that the shorter section of the anode 
wire, $x$, was on the 5-magnet side, and the longer section of the anode wire, L - $x$, was on the 4-magnet side of the wire tension measurement jig. Thus $\mathrm{f}_{1}>\mathrm{f}_{2}$. A straight-forward calculation shows that the generated $e m f$ is linearly proportional to the amplitude of the excitation current, but is proportional to the square of the magnetic field, $B$. Thus the relative heights of the two resonance curves could be used to uniquely determine which resonance was associated with a particular side of the tube, thus eliminating the possibility of not detecting a loose or improperly positioned wire support at its mirror image location, reflected about the mid-length point of the the tube.

Typical resonance curves for each of the four types of drift tubes are shown in Figs. 3a-3d. The constant, frequency-independent offset in each of the four curves is merely an artifact of the input amplitude from the Wavtek function generator, and the voltage offset of the last gain stage of the amplifier output of the true rms $\mathrm{AC}$ to $\mathrm{DC}$ converter. This offset could be trivially removed by a simple adjustment of either of these two devices, or optionally removed in software. However, we chose to explicitly monitor the baseline offset during the use of this system because it was a useful indicator of the overall stability of the system. Under normal operation, the system was very stable on a day-to-day basis. While collecting and displaying the wire tension measurement data in real time, the LSI-11/23 computer also calculated the mean and standard deviation of each of the separate resonant peaks. To speed up the measurement process, the on-line program took relatively large steps in the Wavetek frequency of 0.08 
$-0.12 \mathrm{~Hz}$. Near a resonance, smaller steps of $0.02 \mathrm{~Hz}$ in size were taken, corresponding to 1 LSB on the DAC. A frequency band of $\sim 50$ or $80 \mathrm{~Hz}$ was swept during the course of the wire tension measurement, centered on the $5^{\text {th }}$ harmonic. The width of the frequency band and off-resonance DAC step size used depended on tube type. A typical measurement took $\sim 3.0$ minutes to complete, including installation and removal of the drift tube from the wire tension measurement jig. The on-line program and also the hardware was deliberately made simple, such that personnel unfamiliar with the system could be easily instructed to reliably operate it for drift tube wire tension measurements.

In order to obtain resonance curves that did not contain distortions due to non-linear mechanical or electronic effects, the excitation voltage was set well below an empirically determined critical value. Due to the high sensitivity of this system, we found that it was extremely important not to have any extraneous mechanical or acoustical vibrations present during a wire tension measurement. We also observed that if drift tubes were not rigidly clamped to the wire tension measurement jig, the sensitivity of the wire tension measurement system was such that it could easily detect the induced motion of the drift tube itself, due to the mechanical coupling of the anode wire to the drift tube, which in turn would cause a partial cancellation of the induced emf! This effect was most noticable at the onset of one (or both) of the resonances. This effect graphically emphasized to us the great importance of maintaining pure Dirichlet boundary conditions (i.e. fixed ends of the anode wire) for our system! 
The CDF Central Drift Tube wire tension measurement system was also sensitive enough to detect any systematic variation in the mass per unit length, $\mu$, of the anode wire at the few per cent level. Nominally, a 1000-foot spool of $50 \mu \mathrm{m}$ diameter Stablohm-800 stainless steel wire [7] would be used in stringing a total of $\sim 40-50$ drift tubes. As drift tubes were fabricated in groups of 10 consecutively serial numbered tubes, we routinely histogrammed individual drift tube wire tensions and wave speed $v s$ drift tube serial number to search for any such systematic effects. None were observed at any level of significance.

Fig. 4 shows the wire tension distribution for 2,226 drift tubes fabricated for the CDF Central Drift Tube array. In order to control the tails of this distribution during the wire-stringing stage of drift tube fabrication, we required that drift tubes with less than $T=100$ grams, or more than $T=120$ grams be re-strung. Tubes which did not have their delrin endpin/feedthru assemblies pushed completely into the drift tube during wire-stringing were also restrung. Less than $1 \%$ of all drift tubes made had to be re-strung due to the failure of one (or both) of these criteria. Two people working on this project were responsible for stringing all of the 2,226 drift tubes. Figs. $5 \mathrm{a}$ and $5 \mathrm{~b}$ show the differences in wire tension distribution between the two personnel, as obtained from a subsample of strung tubes. The difference between the two distributions is due to the difference in the degree of stabilization of the wire tensioning weight before the final crimp was made.

The difference in anode wire support location from design for $2,226 \mathrm{drift}$ 
tubes, as measured with the CDF Central Drift Tube wire tension measurement system is shown in Fig. 6. The width of the distribution is entirely consistent with that expected from the intrinsic spatial resolution capabilities associated with the wire tension measurement system. Monitoring this quantity detected 2 out of the 2,226 tubes accidentally strung without wire supports.

The intrinsic frequency resolution of the CDF Central Drift Tube wire tension measurement system for determining the centroids of the two resonant frequencies $f_{1}$ and $f_{2}$ was measured to be $\sigma_{\mathrm{f}} \simeq 0.1 \mathrm{~Hz}$, or $\sigma_{\mathrm{f}} / \mathrm{f} \simeq 0.025 \%$. Thus, the corresponding electronic intrinsic resolution on the drift tube wire tension measurement was measured to be typically $\sigma_{\mathrm{T}} \simeq 0.04 \mathrm{grams}$, of $\sigma_{\mathrm{T}} / \mathrm{T} \simeq 0.04 \%$. The uncertainty in the anode wire length and the absolute frequency calibration of the drift tube wire tension measurement system contribute negligible amounts to the wire tension measurement resolution. However, the $\sigma_{\mu} / \mu \simeq 0.5 \%$ uncertainty in the determination of the mass per unit length of the anode wire, $\mu$ [4] is the dominant source of the overall resolution of the wire tension measurement. Since the wire tension depends linearly on the mass per unit length of the wire, then $\sigma_{\mathrm{T}} \simeq 0.5 \mathrm{grams}$, or $\sigma_{\mathrm{T}} / \mathrm{T} \simeq 0.5 \%$. Note that the intrinsic resolution associated with the determination of the anode wire support position and the wave speed are such that these quantities are not affected by the uncertainty in the determination of the mass per unit length of the anode wire, such that $\sigma_{x} / x \simeq 0.01 \%$ and $\sigma_{v} / v \simeq 0.02 \%$ 


\section{Conclusions}

We have developed a sensitive, computer-controlled method for measuring the anode wire tensions of individual drift tubes which have a wire support located mid-length along the tube. By resonating the anode wire at higher harmonics of the fundamental resonance frequency, simultaneous measurement of the anode wire tension and verification of the location of the wire support was achieved. The anode wire tension for each drift tube was measured to better than $0.5 \%$ accuracy and the location of the anode wire support measured to better than $0.01 \%$ for drift tubes 3 meters in length. We have demonstrated that such a system works extremely well on a large sample of individual drift tubes. Although we chose to operate our system on the $5^{\text {th }}$ harmonic, we could equally as well have chosen to use an even harmonic. This technique can also be used when there is more than one wire support, and also on higher harmonics of the fundamental frequency. By writing wire tension measurement data to a fabrication database, statisical analyses of the wire tensions, wire support locations and other drift tube parameters could be performed, providing invaluable feedback to personnel during the wire-stringing stage of the fabrication of the CDF Central Drift Tube array.

\section{Acknowledgements}

The authors wish to thank H. Scott, F.D. Cogswell, F.W. Witt, R. Spooner, M. Crouch, V.J. Simaitas, D. Lesny and J. Wray for their help and assistance 
in this part of the CDF Central Drift Tube project.

This work was supported in part by the U.S. Department of Energy, contract numbers DE-AC02-76ER01195 (UI) and DE-AC02-76CH03000 (FNAL), seed money from the University of Illinois Department of Physics, the University of Illinois College of Engineering, and a University of Illinois Arnold O. Beckman Research Award from the University of Mlinois Research Board. One of us (S.E.) would also like to gratefully acknowledge the receipt of an Alfred P. Sloan Research Fellowship Award.

\section{References}

[1] S. Bhadra, et al., The Design and Construction of the CDF Central Drif Tube Array, submitted to Nuclear Instruments and Methods, 1987.

[2] Eight distinct wire support locations were in fact used in the CDF Central Drift Tube array. This was achieved simply by rotating one-half of the tubes made with the four principle wire support locations by $180^{\circ}$ (about a perpendicular to the tube axis) during the fabrication of $30^{\circ}$ CDT sectors.

[3] In the initial set-up and debugging phase of the CDT Central Drift Tube wire tension measurement system, we explicitly verified by several different tests that the presence of the wire support decoupled the anode wire into two independent halves.

[4] The lengths of all drift tubes were measured to an accuracy of $\sigma_{\mathrm{L}} \simeq 10 \mu \mathrm{m}$. 
However, because of the design of the drift tube endpin/feedthru assemblies at the ends of the tube, the anode wire lengths were known to an accuracy of $\sigma_{\mathrm{W}} \simeq 100 \mu \mathrm{m}$. The mass per unit length, $\mu=17.3 \pm 0.1 \mathrm{mg} / \mathrm{m}$ of the Stablohm- 800 anode wire was determined by taking several $10 \mathrm{~m}$ length samples and measuring them on a sensitive Mettler AE-100 electronic scale.

[5] M. Atac and M. Mishina, A Sensitive and Simple Method for Measuring Wire Tensions, Fcrmilab Technical Note CDF-133/TM-1125 2562.000 (unpublished), Fermilab, Batavia,IL, 60510 USA.

[6] The design of the true rms AC to DC Module used in the CDF Central Drift Tube wire tension measurement system originated from an article by J.T. Bailey, A True-rms Adapter, in Modern Electronics, p. 56-61, June, 1985, 76 N. Broadway, Hicksville, NY, 11801 USA. We wish to thank H. Scott for bringing this article to our attention. The AD536AJ-D true rms-to-dc converter integrated circuit was obtained from Analog Devices, P.O. Box 280, Norwood, MA, 02062 USA.

[7] The $50 \mu \mathrm{m}$ diameter ultra-finished, stress-relieved, non-magnetic Stablohm800 stainless steel wire was obtained from the California Fine Wire Co., 338 S. Fourth St., P.O. Box 446, Grover City, CA, 93433 USA. 


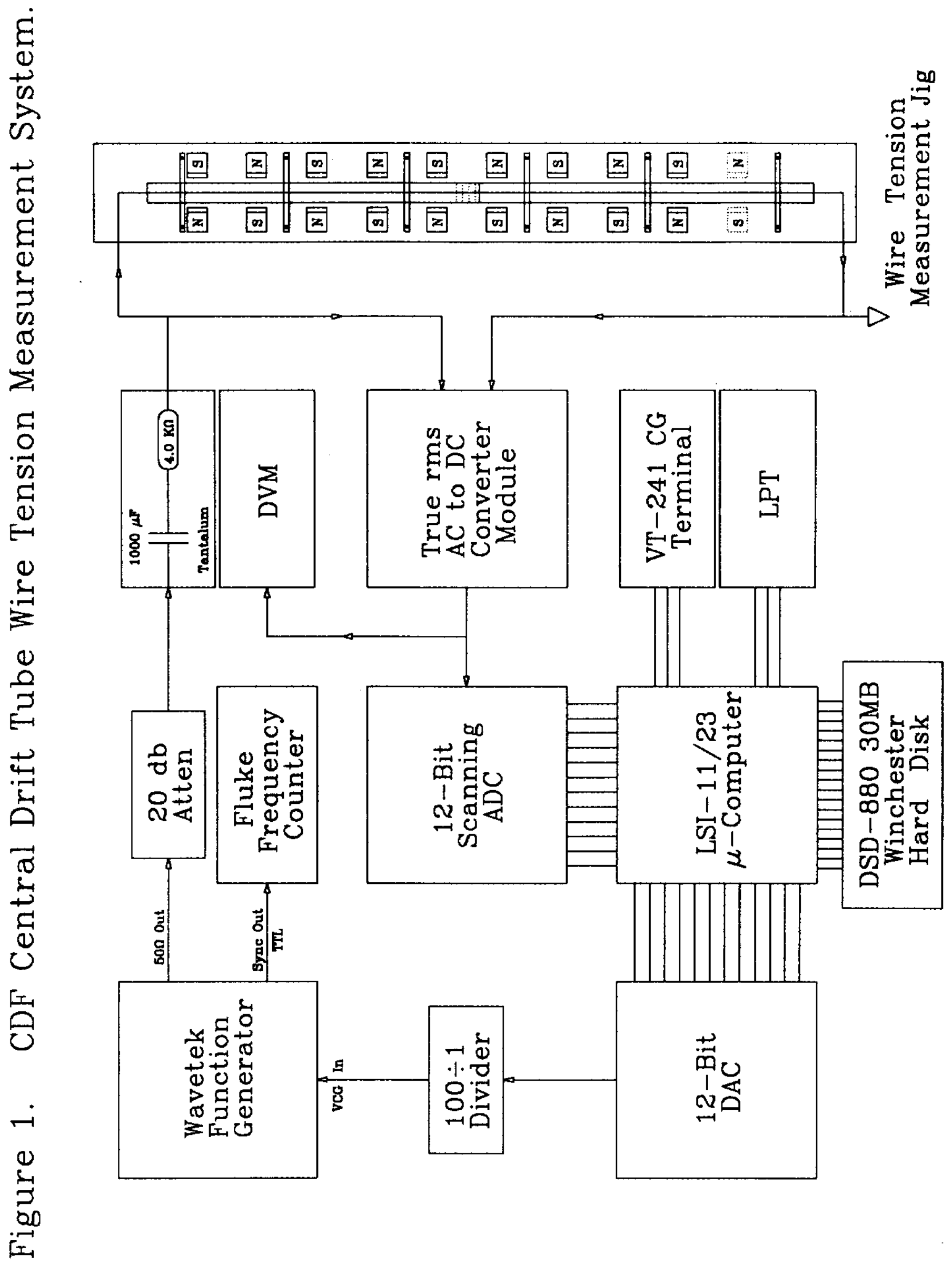



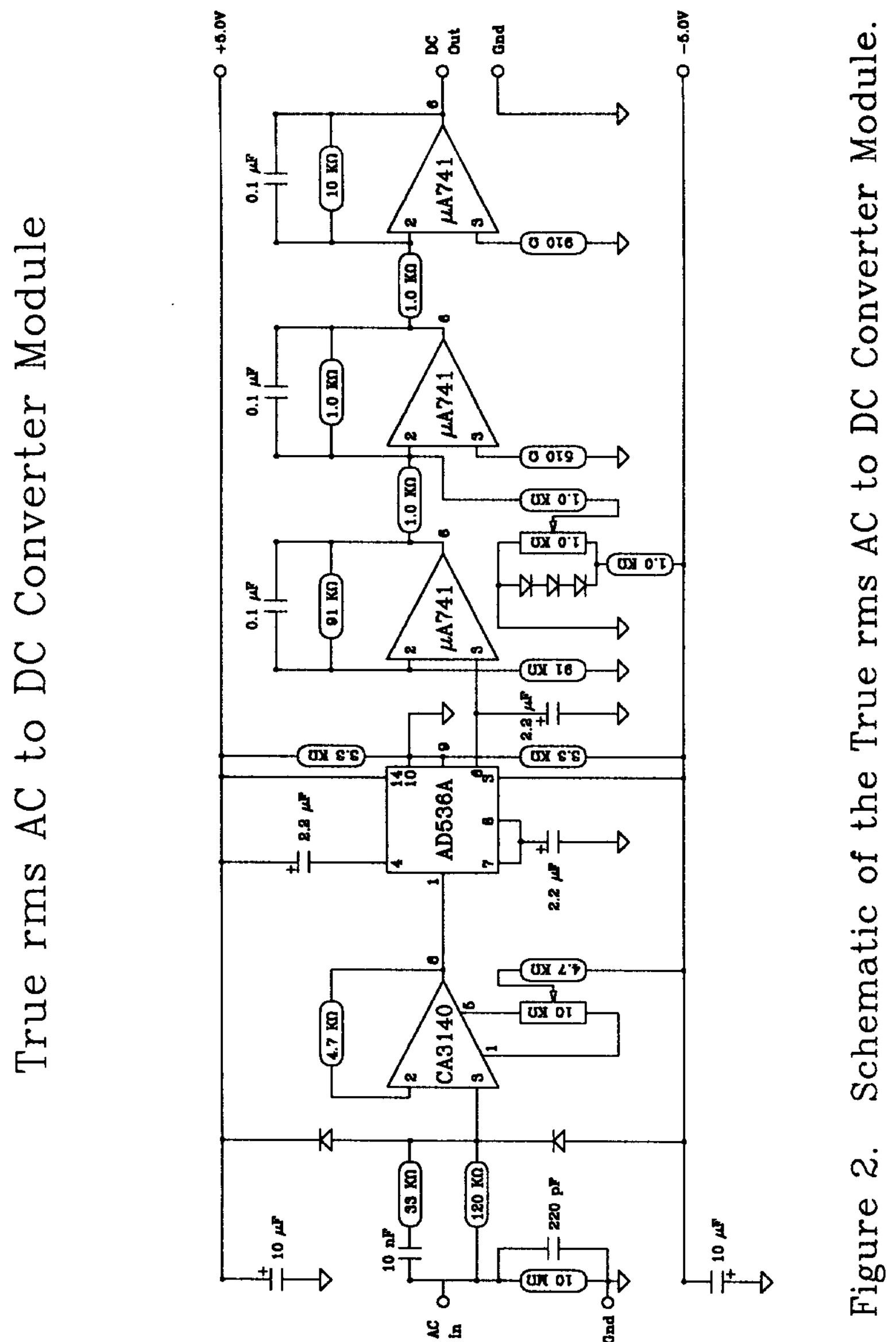

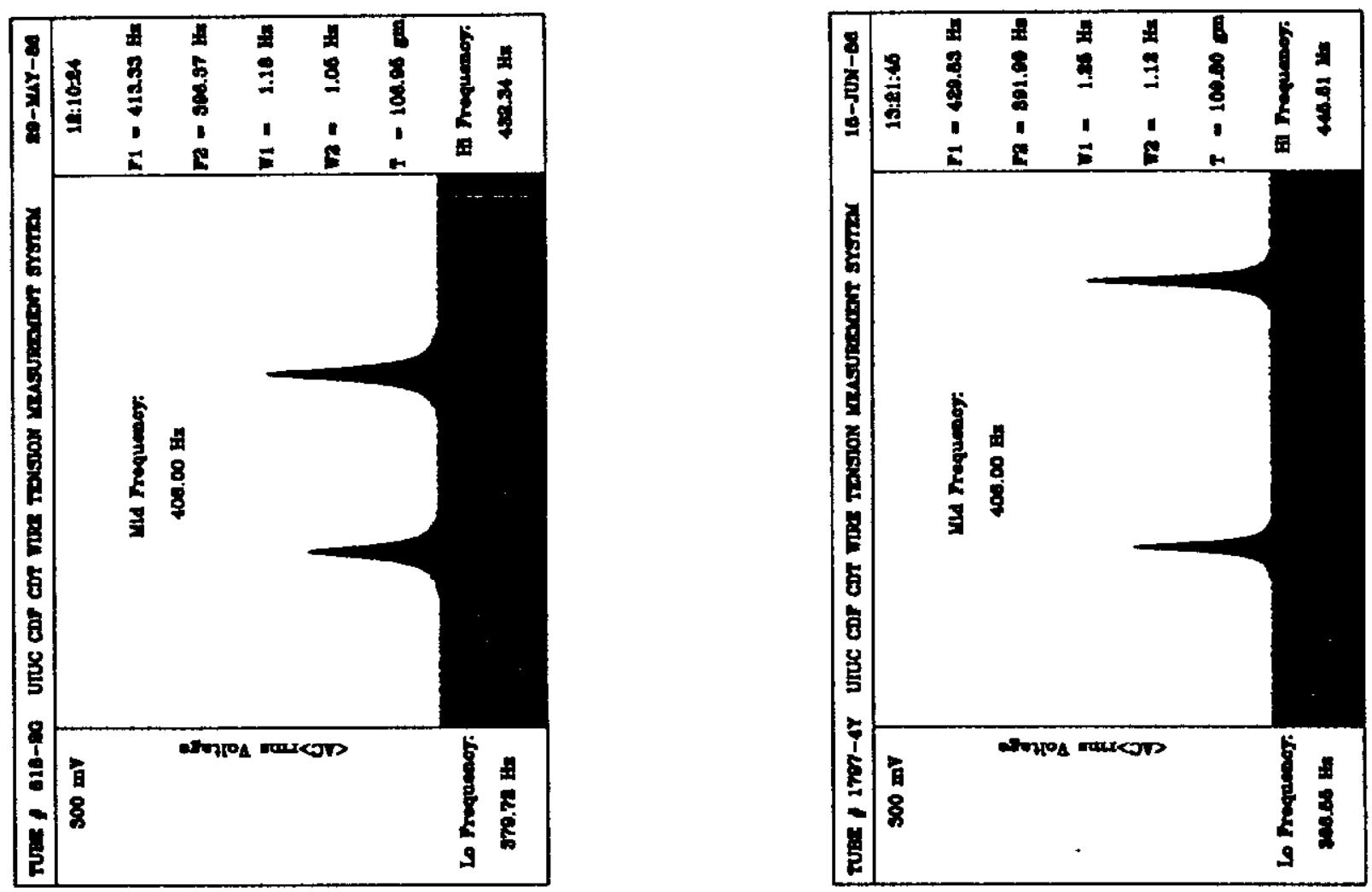

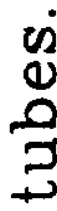

4

4

s

0

त)

븡

¿

n

0
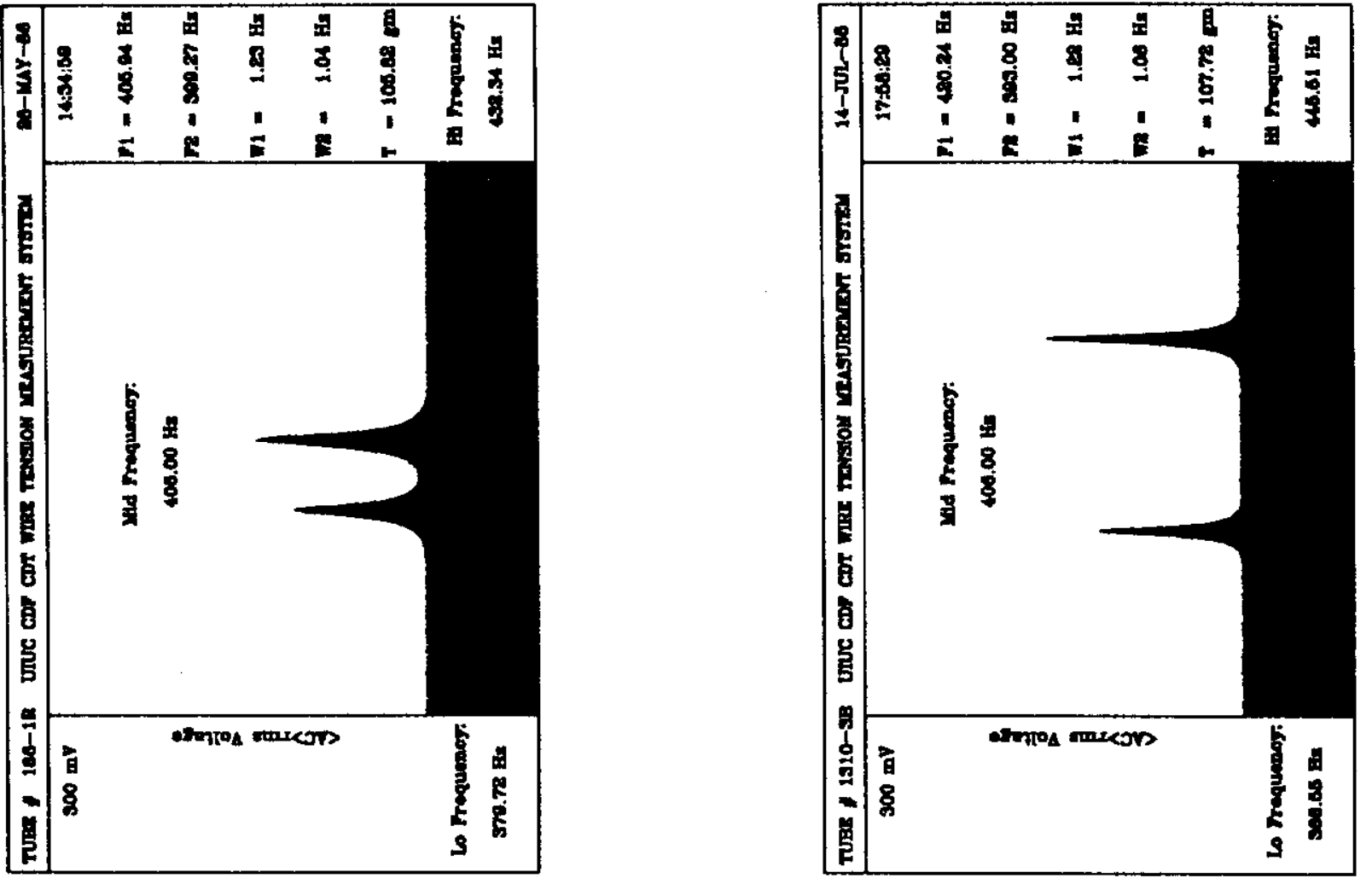

?

0

J

ธ

옹

\&

$\pi$

.

D

$\dot{\sigma}$

0

告 


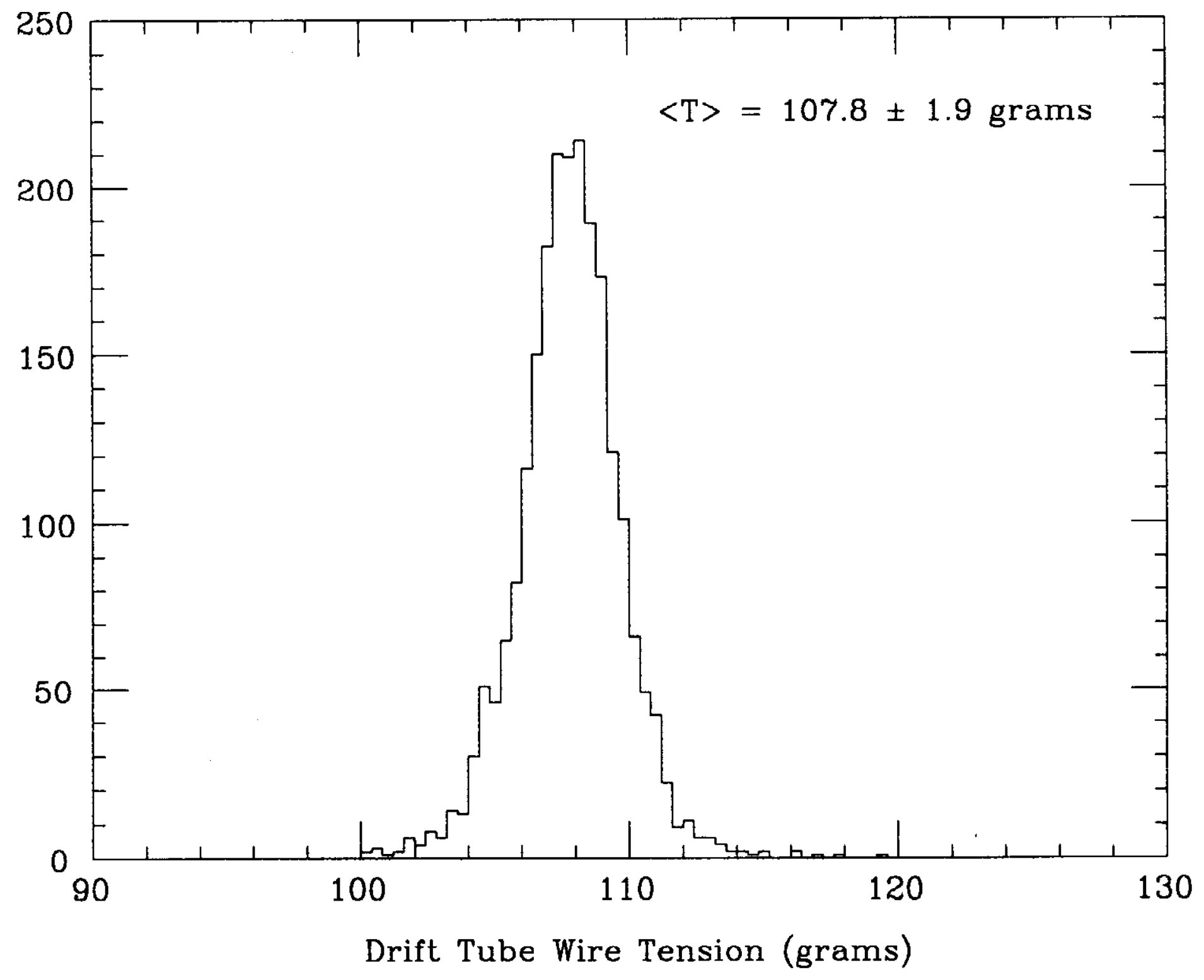

Figure 4. Wire tensions for 2,226 drift tubes. 


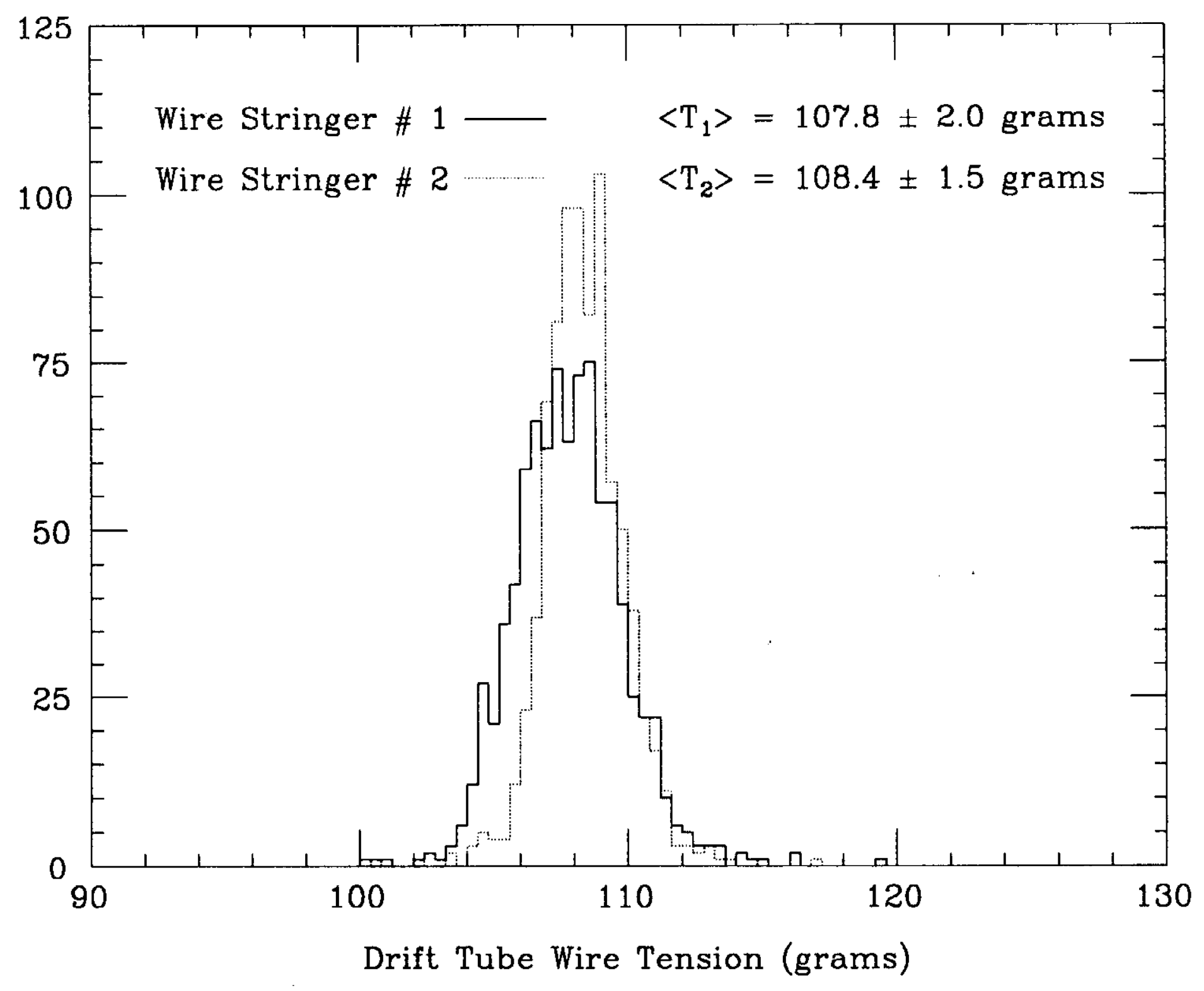

Figure 5. Wire tensions for two different personnel. 


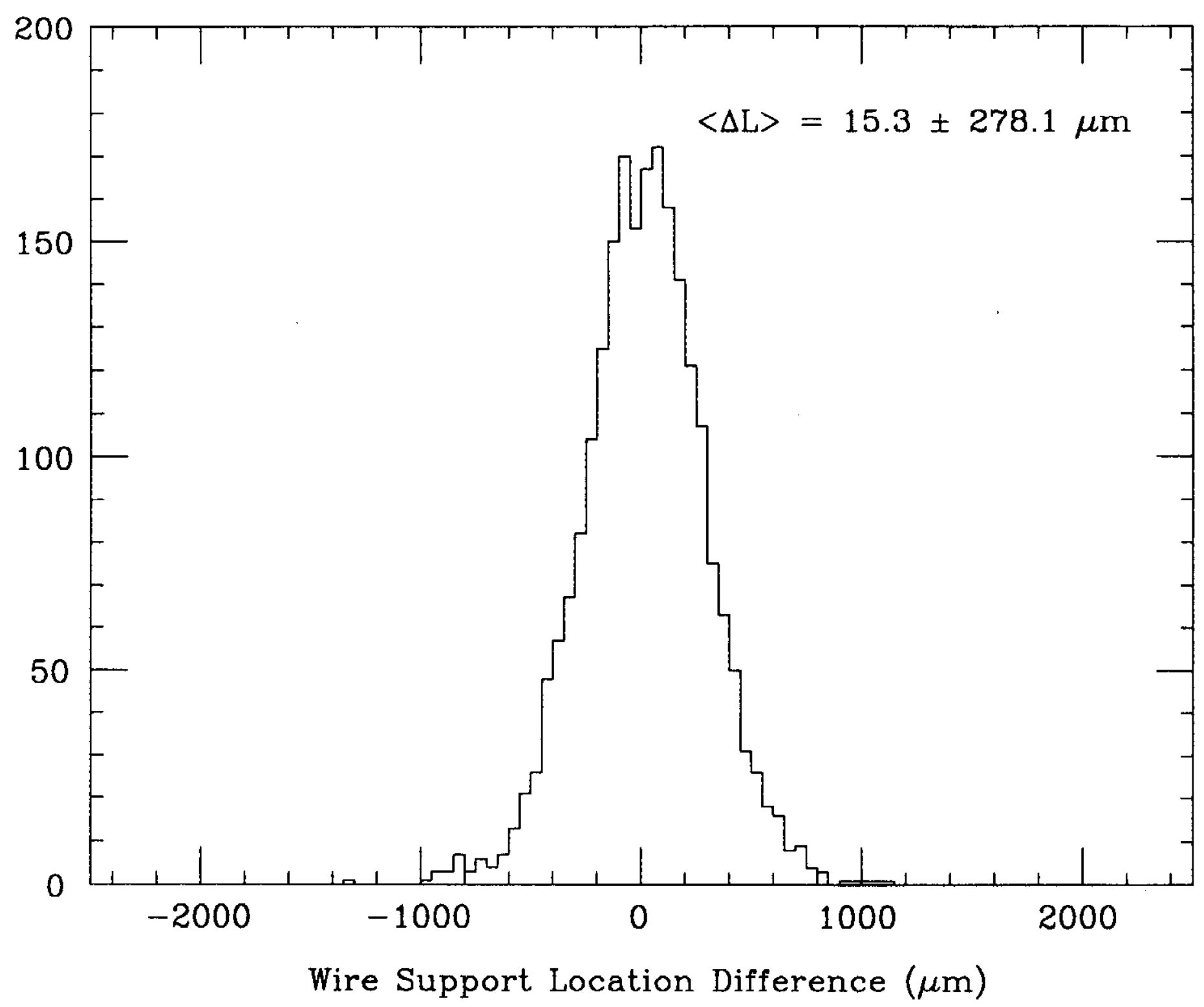

Figure 6. Deviation of wire support locations from design. 